\title{
EGFR Exon 20 Insertion Mutation
}

National Cancer Institute

\section{Source}

National Cancer Institute. EGFR Exon 20 Insertion Mutation. NCI Thesaurus. Code C125605.

Mutation located after the C-helix of the tyrosine kinase domain of EGFR. 